\title{
Proton emission study as a guide to astrophysical rp pro- cess
}

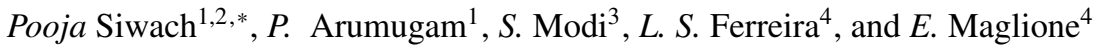 \\ ${ }^{1}$ Department of Physics, Indian Institute of Technology Roorkee, Roorkee 247667, India \\ ${ }^{2}$ Department of Physics, University of Wisconsin-Madison, Madison, Wisconsin 53706, USA \\ ${ }^{3}$ Department of Physics, BIT Sindri, Dhanbad, Jharkhand-828121, India \\ ${ }^{4}$ Centro de Física e Engenharia de Materiais Avançados CeFEMA, and Instituto Superior Técnico, \\ Universidade de Lisboa, Avenida Rovisco Pais, P1049-001 Lisbon, Portugal
}

\begin{abstract}
Proton emitters play an important role in deciding the path of the astrophysical rapid proton capture (rp) process. The lifetime of these nuclei depends on several factors, like the deformation, angular momentum of the emitted proton, residual interaction between valence proton and neutron (especially in case of odd-odd nuclei) and so on. Therefore, it is worth to investigate the structure of proton emitters to understand the rp process path. However, due to lack of data in this exotic region, the theoretical models should be robust and the dependence on the free parameters should be minimal. In this direction, we have developed the first microscopic approach to study the triaxially deformed odd-odd proton emitters. The application of the developed approach to ${ }^{108} \mathrm{I}$, a recently observed proton emitter to investigate the end cycle of the rp process, is discussed.
\end{abstract}

\section{Introduction}

When the limits of the nuclear landscape are crossed on the proton-rich side, a nucleus becomes incapable to hold more protons resulting into spontaneous proton emission. However, due to the high Coulomb barrier, the proton spends enough time inside the nucleus to be measured before decay. Therefore, this phenomenon has been studied a lot both theoretically and experimentally, and provides a unique tool in unveiling the structural properties of nuclei at extreme limits.

The proton emitting nuclei serve as the decisive elements in the path of astrophysical rapid proton capture (rp) process [1]. By investigating the proton separation energies and half-lives one can get direct or indirect information on the path of rp process. For example, with the help of the proton emission half-life of ${ }^{73} \mathrm{Rb}[2]$, the ${ }^{72} \mathrm{Kr}$ was established as a strong waiting point. Furthermore, the information of proton separation energy of ${ }^{109} \mathrm{I}$ along with the $\alpha$-decay energy [3] assisted the indirect measurement of proton separation energy of ${ }^{105} \mathrm{Sb}$ to investigate the possible paths in the end-cycle ( $\mathrm{Sn}-\mathrm{Sb}$-Te cycle) of rp process.

Recently, in an experiment performed at Argonne National Laboratory, a weak proton emission branch was detected in ${ }^{108}$ I (predominantly an alpha emitter) [4]. This study revealed that the rp process has to deviate its path from ${ }^{106} \mathrm{Sb}$ due to its proton unboundedness.

\footnotetext{
*e-mail: psiwach@physics.wisc.edu
} 
This study further resolved the ambiguities in the path of rp process end cycle. However, to investigate the structural properties of ${ }^{108} \mathrm{I}$, we need a theoretical approach which is less reliant on the freely adjustable parameters, and can incorporate the triaxiality and residual neutron-proton (np) interaction in a reliable way. Therefore, we have developed a nonadiabatic quasiparticle approach [5-7] for triaxial odd-odd proton emitters, satisfying the above requirements.

\section{Formalism}

We treat the odd-odd nuclei as the valence proton and neutron coupled to the even-even core. For the valence proton and neutron, we consider the nuclear mean-field potential in the Woods-Saxon potential form. A very detailed formalism is given elsewhere [6 -8]. In the present work, we incorporate the residual $\mathrm{np}$ interaction $V_{n p}$ in the zero-range interaction form given by [6]

$$
V_{n p}=-\sqrt{\frac{8 \pi^{3}}{v^{3}}} W\left[(1-\alpha)+\alpha \overrightarrow{\sigma_{p}} \cdot \overrightarrow{\sigma_{n}}\right] \delta\left(\overrightarrow{r_{p}}-\overrightarrow{r_{n}}\right),
$$

where $\sigma_{p}$ and $\sigma_{n}$ are Pauli spin matrices, $\alpha$ and $W$ are the strength parameters, and $v$ is the frequency related to the size of the nucleus. The total Hamiltonian of this system is solved to obtain the parent (before decay) wave function. In this microscopic approach, we calculate the overlap of initial (parent) and final (daughter nucleus+proton) wave functions. The partial decay width can be written as

$$
\begin{aligned}
\Gamma_{l_{p} j_{p}}^{I I_{d}}= & \left.\frac{\hbar^{2} k}{\mu}\left(\frac{2 I_{d}+1}{2 I+1}\right)\right|_{j_{p} \Omega_{p} j_{n} \Omega_{n} K_{d} \Omega K} A_{j_{n} \Omega_{n} K_{d}}^{I_{d}} a_{\Omega_{p} \Omega_{n} \Omega K}^{I}\left(\left\langle I_{d} K_{d} j_{p} \Omega_{p} \mid I K\right\rangle\right. \\
& \left.+(-1)^{I_{d}-K_{d}}\left\langle I_{d}-K_{d} j_{p} \Omega_{p} \mid I K\right\rangle\right)\left.u_{\Omega_{p}} N_{l_{p} j_{p}}\right|^{2}
\end{aligned}
$$

where $I$ and $I_{d}$ are the angular momentum of parent and daughter nucleus, respectively, and $K$ and $K_{d}$ are their projection on 3-axis. $a$ and $A$ are the mixing coefficients of the parent and daughter wave functions, respectively. $j_{i}$ and $\Omega_{i}(i=p, n)$ are the quantum numbers of proton and neutron. Square of $u_{\Omega_{p}}$ gives the probability of orbital to be empty in the parent nucleus. The term in angular brackets represents the Clebsch-Gordon coefficients. The asymptotic normalisation constant is given by

$$
N_{l_{p} j_{p}}^{\Omega_{p}}=\frac{\phi_{l j}^{\Omega_{p}}(\mathcal{R})}{G_{l}(k \mathcal{R})+\iota F_{l}(k \mathcal{R})},
$$

where $\phi$ is the proton wave function at distance $\mathcal{R}$. $G$ and $F$ are the irregular and regular parts of the Coulomb wave function.

The total decay width can be obtained by summing over all the states as

$$
\Gamma^{I I_{d}}=\sum_{j_{p}=\left|I-I_{d}\right|}^{I+I_{d}} \Gamma_{l_{p} j_{p}}^{I I_{d}}
$$

which gives the half-life $T_{1 / 2}=\hbar \ln 2 / \Gamma^{I I_{d}}$. 

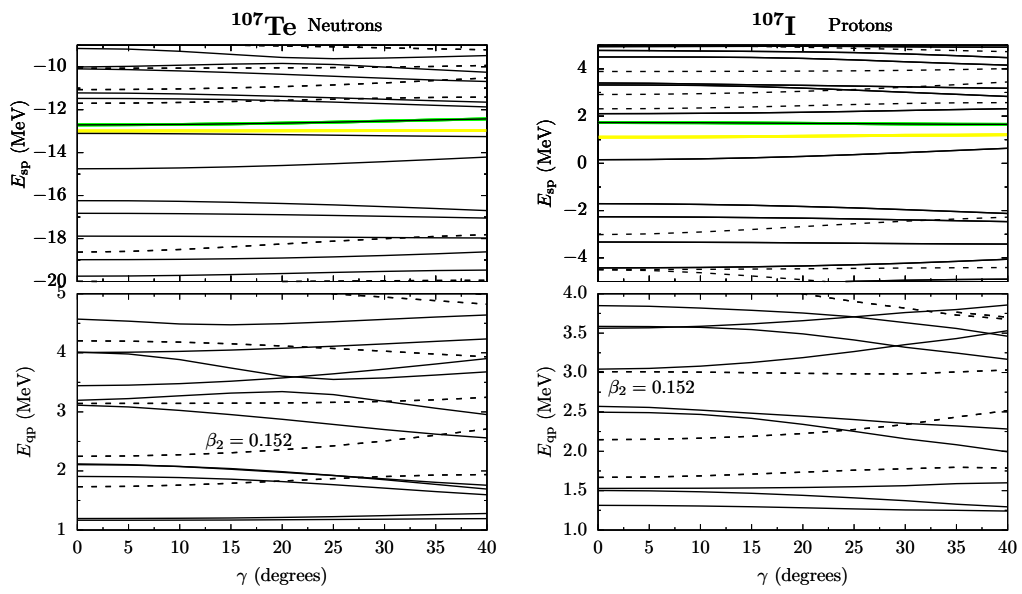

Figure 1. Single-particle (top) and quasiparticle (bottom) energies of neutrons in ${ }^{107} \mathrm{Te}$ (left) and protons in ${ }^{107} \mathrm{I}$ (right) at $\beta_{2}=0.152$ as a function of $\gamma$. Positive and negative parity levels are represented by the solid and dashed lines, respectively. Green and yellow lines represent the Fermi level and the chemical potential from BCS calculations, respectively.

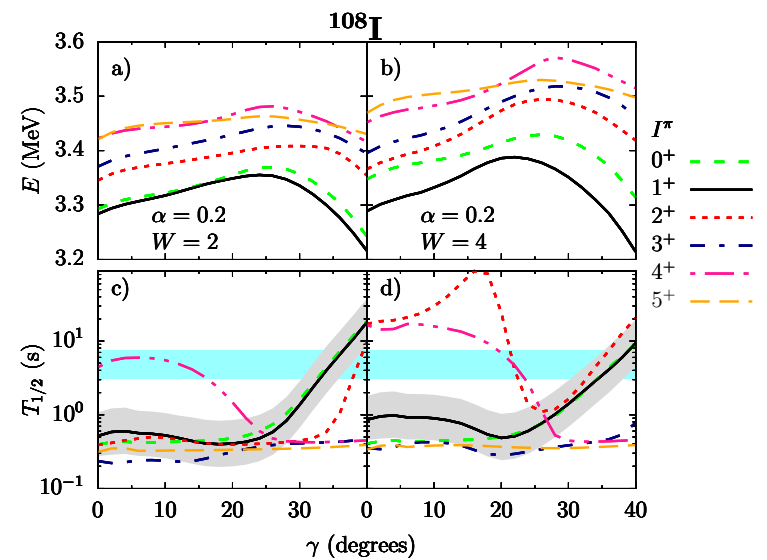

Figure 2. Rotational energies (panels $\mathrm{a}$ and $\mathrm{b}$ ) and proton emission half-lives (panels $\mathrm{c}$ and d) at $\beta_{2}=0.152$ as a function of $\gamma$ for different set of parameters $\alpha$ and $W$ of residual np interaction. Gray width in half-life represents the uncertainties due to the one in measured $Q_{p}$ value.

\section{Results and discussion}

To calculate the proton emission half-life, we need the information on the ground state of daughter nucleus $\left({ }^{107} \mathrm{Te}\right)$, which is found to be $5 / 2^{+}$[7]. Considering the axial deformation $\left(\gamma=0^{\circ}\right)$, the measured data could not be reproduced. Therefore, we incorporate the triaxiality (with non-zero $\gamma$ ), and the resulting single-particle and quasiparticle energies for neutrons in ${ }^{107} \mathrm{Te}$ and protons in ${ }^{107} \mathrm{I}$ are given in Fig. 1. We notice that with increase in $\gamma$, the singleparticle and quasiparticle energies for neutrons come closer to the Fermi level. Similarly, for protons in ${ }^{107} \mathrm{I}$, the quasiparticle energies reveal an interesting variation at large $\gamma$ with one level coming very close to the Fermi level. We see the impact of these variations in the proton emission half-lives as discussed in the forthcoming discussion.

We proceed to investigate the properties of the ${ }^{108} \mathrm{I}$. We observe from the rotational energies shown in Fig. 2 2 that the $0^{+}$and $1^{+}$are the lowest in energy whereas with increasing the strength of residual np interaction, $1^{+}$is the more favoured level. The proton emission half- 
life of this level reproduces the measured one at $\gamma \approx 30^{\circ}$. Other levels crossing the measured half-life at a lower $\gamma$ are very high in energy. Hence, we conclude that the proton emission occurs from the $1^{+}$level in ${ }^{108} \mathrm{I}$ and it is highly triaxial with $\gamma \approx 30^{\circ}$. These conclusions agree with the ones obtained with the residual $\mathrm{np}$ interaction in the constant potential form [7].

\section{Acknowledgements}

This work was supported in part by the U.S. Department of Energy, Office of Science, Office of High Energy Physics, under Award No. DE-SC0019465.

\section{References}

[1] H. Schatz, A. Aprahamian, J. Görres, M. Wiescher, T. Rauscher, J. Rembges, F.K. Thielemann, B. Pfeiffer, P. Moller, K.L. Kratz et al., Phys. Rep. 294, 167 (1998)

[2] H. Suzuki, L. Sinclair, P.A. Söderström, G. Lorusso, P. Davies, L.S. Ferreira, E. Maglione, R. Wadsworth, J. Wu, Z.Y. Xu et al., Phys. Rev. Lett. 119, 192503 (2017)

[3] C. Mazzocchi, R. Grzywacz, S.N. Liddick, K.P. Rykaczewski, H. Schatz, J.C. Batchelder, C.R. Bingham, C.J. Gross, J.H. Hamilton, J.K. Hwang et al., Phys. Rev. Lett. 98, 212501 (2007)

[4] K. Auranen, D. Seweryniak, M. Albers, A. Ayangeakaa, S. Bottoni, M. Carpenter, C. Chiara, P. Copp, H. David, D. Doherty et al., Phys. Lett. B 792, 187 (2019)

[5] G. Fiorin, E. Maglione, L.S. Ferreira, Phys. Rev. C 67, 054302 (2003)

[6] P. Siwach, P. Arumugam, S. Modi, L.S. Ferreira, E. Maglione, J. Phys. G: Nucl. Part. Phys. 47, 125105 (2020)

[7] P. Siwach, P. Arumugam, S. Modi, L.S. Ferreira, E. Maglione, Phys. Rev. C 103, L031303 (2021)

[8] P. Siwach, Ph.D. thesis, Indian Institute of Technology Roorkee (2021) 